\title{
THE LATTICE THEORY OF POST ALGEBRAS
}

\author{
BY \\ GEORGE EPSTEIN
}

1. Introduction. The algebra corresponding to the $n$-valued logic described by E. L. Post [7] in 1921 was first developed by P. C. Rosenbloom [9] in 1942. Rosenbloom's axioms are based on a minimum of undefined operations and are therefore quite complicated. This complexity also hinders his development of the theory. In this paper, a set of axioms for Post algebras is presented which makes use of a greater number of operations, as well as certain constants. These operations $C_{0}, \cdots, C_{n-1}$ are generalized complementation operators, where $n$ is the order of the Post algebra. The axioms, given in terms of these operators, are very simple. In addition, the simplicity of the operations makes a large part of the theory much more transparent. Another striking feature of the development is the role played by the underlying Boolean algebra of the Post algebra. The existence of this Boolean algebra has been known for a long time, but this fact has not been as fully exploited as in this approach. It will be shown, for example, that the representation theory for Post algebras follows immediately from the corresponding theory for Boolean algebras. No further use of the Axiom of Choice is needed. In addition many properties of a Post algebra, such as completeness, infinite distributivity, and the atomistic property, are fully mirrored by the corresponding properties for the underlying Boolean algebra.

The notation is explained in $\S 2$. $\S 3$ presents the axioms, and various theorems and remarks concerning the arithmetic and structure of the algebra. $\S 4$ discusses Post functions and their reduction to a given form. Examples are given in $\S 5$. The representation theory is described in $\S 6$, and $\S 7$ discusses completeness properties of infinite Post algebras.

2. Notation. The usual lattice notation is employed. The supremum of $x$ and $y$ is denoted by $x \vee y$, and the infimum of $x$ and $y$ is denoted by $x \wedge y$, or more briefly, by $x y$. The symbols $\bigvee_{x_{i}}$ and $\Lambda x_{i}$ denote, respectively, the supremum and infimum of the $x_{i}$ over a specified index set. The symbols $\bigvee_{L ;} x_{i}$ and $\Lambda_{L ;} x_{i}$ emphasize that the supremum or infimum is taken in the lattice $L$. If $x$ has a complement, it is denoted by $\{x\}$ - or, if convenient, by $\bar{x}$.

3 . Formulation. Let $n$ be a fixed integer satisfying $n \geqq 2$. Let $L$ be a distributive lattice with zero 0 and unit $u$, and satisfying the following conditions:

Axiom 1. For every element $x \in L$ there exist $n$ elements $C_{0}(x), C_{1}(x), \cdots$, $C_{n-1}(x)$ which are pairwise disjoint and whose supremum is $u$; that is, $C_{i}(x) C_{j}(x)=0$ for $i \neq j$ and $\bigvee_{i=0}^{n-1} C_{i}(x)=u$.

Presented to the Society, June 20, 1959; received by the editors April 24, 1959. 
Axiom 2. There exist $n$ fixed elements of $L$, denoted $0=e_{0}, e_{1}, \cdots, e_{n-2}$, $e_{n-1}=u$ with the properties:

2a. The elements form a chain, with $e_{i-1} \leqq e_{i}$ for $1 \leqq i \leqq n-1$.

2b. If $x \in L$ and $x e_{1}=0$, then $x=0$.

2c. If $x \in L$ and, for some $i, x \bigvee e_{i-1}=e_{i}$, then $x=e_{i}$.

AxIom 3. For every $x \in L, x=\mathrm{V}_{i=0}^{n-1} e_{i} C_{i}(x)$; that is,

$$
x=e_{1} C_{1}(x) \vee e_{2} C_{2}(x) \vee \cdots \vee e_{n-2} C_{n-2}(x) \vee C_{n-1}(x) .
$$

It will be shown in $\S 6$ that $L$ is a Post algebra and, conversely, every Post algebra is such a lattice. The remaining work in this section is an exposition of fundamental consequences.

Lemma 1. If $x \in L$ and $x e_{i}=0$ for some $i$ in the range $1 \leqq i \leqq n-1$, then $x=0$.

Proof. If $x e_{i}=0$, then $\left(x e_{i}\right) e_{1}=0$. Hence $x e_{1}=0$ by Axiom $2 \mathrm{a}$ and $x=0$ by Axiom 2b.

LeMmA 2. If $x \in L$ and if $x \bigvee e_{i}=e_{j}$ for some $i$ and $j$, with $i<j$, then $x=e_{j}$.

Proof. If $x \bigvee e_{i}=e_{j}$, then $x \bigvee e_{i} \bigvee e_{j-1}=e_{j} \bigvee e_{j-1}$, and $x \bigvee e_{j-1}=e_{j}$ by Axiom 2a. Hence $x=e_{j}$ by Axiom 2c.

It is well known that the complemented elements of a distributive lattice form a sublattice which is a Boolean algebra. This leads to the following definition.

Definition 1. Let $B$ be the Boolean algebra of complemented elements of $L$.

Theorem 1. If $x \in L$, then $x \in B$ if and only if $x=C_{i}(y)$ for some $i$ and some $y \in L$.

Proof. If $x=C_{i}(y)$, then $\bar{x}=\mathrm{V}_{j \neq i} C_{j}(y)$ by Axiom 1. On the other hand, suppose $x$ has a complement $\bar{x}$. Then $x \leqq e_{n-2} \vee C_{n-1}(x)$ from Axiom 3, so $e_{n-2} \bigvee \bar{x} \bigvee C_{n-1}(x)=u$. Hence $\bar{x} \bigvee C_{n-1}(x)=u$, by Lemma 2 , and therefore $x \leqq C_{n-1}(x)$. Since $x \geqq C_{n-1}(x)$ by Axiom 3, it follows that $x=C_{n-1}(x)$.

Lemma 3. If $b \in B$ and $b e_{i}=b e_{j}$ for some $i$ and $j$ with $i<j$, then $b=0$.

Proof. Using Axiom 2a, $e_{j}=e_{j} b \bigvee e_{j} b=e_{j} b \bigvee e_{i} b \leqq e_{j} b \bigvee e_{i} \leqq e_{j}$, so that $b e_{j} \vee e_{i}$ $=e_{j}$. Hence $b e_{j}=e_{j}$ by Lemma 2 . Thus $b e_{j}=b\left(b e_{j}\right)=0$, and, by Lemma $1, b=0$.

Theorem 2. (Uniqueness Theorem). The $C_{i}(x)$ are unique. That is, for any given $x \in L$, there is only one sequence of elements $C_{0}(x), C_{1}(x), \cdots$, $C_{n-1}(x)$ satisfying Axiom 1 and Axiom 3.

Proof. Suppose there is another such sequence, say $C_{0}^{\prime}(x), C_{1}^{\prime}(x), \cdots$, $C_{n-1}^{\prime}(x)$. By Axiom 3, $\bigvee_{k=0}^{n-1} e_{k} C_{k}(x)=\bigvee_{k=0}^{n-1} e_{k} C_{k}^{\prime}(x)$. Thus if $i \neq j$, then $C_{i}(x) C_{j}^{\prime}(x) \bigvee_{k=0}^{n-1} e_{k} C_{k}(x)=C_{i}(x) C_{j}^{\prime}(x) \bigvee_{k=0}^{n-1} e_{k} C_{k}^{\prime}(x)$, and, by Axiom 1 , $e_{i} C_{i}(x) C_{j}^{\prime}(x)=e_{j} C_{i}(x) C_{j}^{\prime}(x)$. Since $C_{i}(x) C_{j}^{\prime}(x) \in B$, Lemma 3 shows that 
$C_{i}(x) C_{j}^{\prime}(x)=0$. The result now follows from Axiom 1, for using the fact that $u=\mathrm{V}_{\mathbf{k}=0}^{n-1} C_{k}(x)$, for every $j, C_{j}^{\prime}(x)=C_{j}^{\prime}(x) \bigvee_{k=0}^{n-1} C_{k}(x)$, and $C_{j}^{\prime}(x)=C_{j}^{\prime}(x) C_{j}(x)$. Likewise $u=\mathrm{V}_{k=0}^{n-1} C_{k}^{\prime}(x)$, so that $C_{j}(x)=C_{j}(x) \bigvee_{k=0}^{n-1} C_{k}^{\prime}(x)=C_{j}(x) C_{j}^{\prime}(x)$. Therefore $C_{j}(x)=C_{j}^{\prime}(x)$ for each $j$, and the proof is complete.

THEOREM 3. For each $i$ in the range $0 \leqq i \leqq n-1$,

$$
\begin{aligned}
C_{n-1}\left(C_{i}(x)\right) & =C_{i}(x), \\
C_{j}\left(C_{i}(x)\right) & =0,
\end{aligned}
$$$$
\text { for } 0<j<n-1 \text {, }
$$

and

$$
C_{0}\left(C_{i}(x)\right)=\underset{k \neq i}{\bigvee} C_{k}(x)=\left\{C_{i}(x)\right\}-.
$$

Proof. For given $i$, the $n$ elements $\mathrm{V}_{k \neq i} C_{k}(x), 0,0, \cdots, 0, C_{i}(x)$ are pairwise disjoint and their supremum is $u$, so that Axiom 1 is satisfied. Axiom 3 is satisfied since $C_{i}(x)=e_{1} 0 \vee \cdots \vee e_{n-2} 0 \vee C_{i}(x)$. Hence the result follows by the uniqueness theorem. $=b$.

Corollary. If $b \in B$, then $C_{0}(b)=\bar{b}, C_{j}(b)=0$ for $0<j<n-1$, and $C_{n-1}(b)$

Proof. This follows from Theorem 1.

Theorem 4. If $i \neq j$, then $C_{i}\left(e_{j}\right)=0$, and $C_{i}\left(e_{i}\right)=u$. The elements $e_{i}$, $i$ $=0,1, \cdots, n-1$ are distinct and unique.

Proof. The first part of the theorem is again an immediate consequence of the uniqueness theorem. If $i \neq j$ and $e_{i}=e_{j}$, then $C_{i}\left(e_{i}\right)=u$ and $C_{i}\left(e_{j}\right)=C_{j}\left(e_{j}\right)$ $=u$, contradicting Axiom 1. If there is another sequence of elements $0, e_{1}^{\prime}, \cdots, e_{n-2}^{\prime}, u$ satisfying Axiom 2 and Axiom 3, then for every $x \in L$ $x=\mathrm{V}_{k=0}^{n-1} e_{k}^{\prime} C_{k}(x)$, so that for $x=e_{i}, i=1,2, \cdots, n-2, e_{i}=e_{i}^{\prime}$. Thus the elements $e_{i}, i=0,1, \cdots, n-1$ are both unique and distinct.

Theorem 5. The lattice $L$ is pseudo-complemented; that is, if $x \in L$, there exists $x^{*} \in L$ such that $x y=0$ if and only if $y \leqq x^{*}$.

Proof. It is clear that $x^{*}=C_{0}(x)$, for by Axiom 3 and Axiom $1 x C_{0}(x)=0$, and $x y=0$ if $y \leqq C_{0}(x)$. Conversely, if $x y=0$, then $e_{k} C_{k}(x) y=0$ for each $k$, since $y \bigvee_{k=1}^{n-1} e_{k} C_{k}(x)=0$. Hence $C_{k}(x) y=0$ for each $k \geqq 1$ by Lemma 1 . Thus $y \bigvee_{k=1}^{n-1} C_{k}(x)=y\left\{C_{0}(x)\right\}^{-}=0$, and $y \leqq C_{0}(x)$.

Theorem 6. If $b_{i} \in B$ and $x=\bigvee_{i=1}^{n-1} e_{i} b_{i}$, then $x=\bigvee_{i=1}^{n-1} e_{i}\left(\bigvee_{j=1}^{n-1} b_{j}\right)$, and

$$
C_{0}(x)=\bigwedge_{j=1}^{n-1} b_{j}, \quad C_{i}(x)=b_{i} \bigwedge_{j=i+1}^{n-1} b_{j} \quad \text { for } i=1, \cdots, n-2,
$$

and $C_{n-1}(x)=b_{n-1}$. 
Proof. If $x=\mathrm{V}_{j=1}^{n-1} e_{j} b_{j}$, then

$$
x=\bigvee_{j=1}^{n-1}\left(\bigvee_{i=1}^{j} e_{i}\right) b_{j}=\bigvee_{i=1}^{n-1} e_{i}\left(\bigvee_{j=i}^{n-1} b_{j}\right)
$$

It is clear that

$$
\bigvee_{i=k}^{n-2}\left(b_{i}\left(\bigwedge_{j=i+1}^{n-1} b_{j}\right)\right) \bigvee b_{n-1}=\bigvee_{i=k}^{n-1} b_{i} \quad \text { for } 0<k<n-1
$$

For brevity, let $c_{i}=b_{i}\left(\bigwedge_{j=i+1}^{n-1} b_{j}\right), 0<i<n-1$, and set $c_{n-1}=b_{n-1}$. Then

$$
x=\bigvee_{i=1}^{n-1} e_{i}\left(\bigvee_{j=i}^{n-1} b_{j}\right)=\bigvee_{i=1}^{n-1} e_{i}\left(\bigvee_{j=i}^{n-1} c_{j}\right)=\bigvee_{j=1}^{n-1} c_{j}\left(\bigvee_{i=1}^{j} e_{i}\right)=\bigvee_{j=1}^{n-1} e_{j} c_{j}
$$

Since the $n$ elements $\Lambda_{i=1}^{n-1} b_{i}, c_{1}, \cdots, c_{n-1}$ are pairwise disjoint and their supremum is $u$, the uniqueness theorem yields the final result.

The following definition and consequent theorems are preliminary to the work of $\S 7$. They are also used to give simple proofs of Theorem 10 and Theorem 12.

Definition 2. Let $D_{i}(x)=\mathrm{V}_{j=i}^{n-1} C_{j}(x), i=1,2, \cdots, n-1$.

Clearly $D_{i}(x) \geqq D_{j}(x) \in B$ for all $x \in L$ and all $i$ and $j$ in the range $1 \leqq i \leqq j$ $\leqq n-1$. Axiom 3 and Theorem 6 show that $x=\bigvee_{i=1}^{n-1} e_{i} D_{i}(x)$. The following theorem is another consequence of Theorem 6 .

TheOREM 7. If $b_{i} \geqq b_{j} \in B$ for all $i$ and $j$ in the range $1 \leqq i \leqq j \leqq n-1$, then $b_{i}=D_{i}(x)$, where $x=\bigvee_{i=1}^{n-1} e_{i} b_{i}$.

Proof. Using Theorem 6, $D_{k}(x)=\mathrm{V}_{j=k}^{n-1} C_{j}(x)=\mathrm{V}_{j=k}^{n-1} b_{j}=b_{k}$.

THEOREM 8. For each $i=1,2, \cdots, n-1$ and for every $x \in L$ and $y \in L$, $D_{i}(x \bigvee y)=D_{i}(x) \bigvee D_{i}(y)$.

Proof. Since $D_{i}(x) \geqq D_{j}(x) \in B$ and $D_{i}(y) \geqq D_{j}(y) \in B$ for all $i$ and $j$ in the range $1 \leqq i \leqq j \leqq n-1, D_{i}(x) \bigvee D_{i}(y) \geqq D_{j}(x) \bigvee D_{j}(y) \in B$. Since

$$
x \bigvee y=\bigvee_{i=1}^{n-1} e_{i} D_{i}(x) \bigvee \bigvee_{i=1}^{n-1} e_{i} D_{i}(y)=\bigvee_{i=1}^{n-1} e_{i}\left(D_{i}(x) \bigvee D_{i}(y)\right),
$$

the result follows from Theorem 7 .

TheOREM 9. A necessary and sufficient condition that $x \leqq y$ is that $D_{i}(x)$ $\leqq D_{i}(y)$ for each $i=1, \cdots, n-1$.

Proof. If $x \leqq y$, then $x \bigvee y=y$ and $D_{i}(x) \bigvee D_{i}(y)=D_{i}(y)$ by Theorem 8; that is, $D_{i}(x) \leqq D_{i}(y)$ for each $i=1,2, \cdots, n-1$. Conversely, if $D_{i}(x)$ $\leqq D_{i}(y)$ for each $i=1,2, \cdots, n-1$, then 


$$
x=\bigvee_{i=1}^{n-1} e_{i} D_{i}(x) \leqq \bigvee_{i=1}^{n-1} e_{i} D_{i}(y)=y
$$

THEOREM 10 (DUALITY THEOREM). The lattice $L$ is dually isomorphic with itself under the mapping $\beta(x)=\bigvee_{i=1}^{n-1} e_{i}\left\{D_{n-i}(x)\right\}-$. If $L^{\prime}$ denotes the dual lattice and $C_{i}^{\prime}, e_{i}^{\prime}$ are the operators and elements of Axiom 1 and Axiom 2 for $L^{\prime}$, while $D_{i}^{\prime}$ are the operators of Definition 2 for $L^{\prime}$, then

$$
\begin{aligned}
e_{i}^{\prime} & =e_{n-i-1}, \\
C_{i}^{\prime}(x) & =\left\{C_{n-i-1}(x)\right\}-
\end{aligned}
$$

and

$$
D_{i}^{\prime}(x)=D_{n-i}(x) .
$$

Proof. Since $\left\{D_{n-i}(x)\right\}-\geqq\left\{D_{n-j}(x)\right\}-\in B$ for all $i$ and $j$ in the range $1 \leqq i \leqq j \leqq n-1$, Theorem 7 yields

$$
D_{i}(\beta(x))=\left\{D_{n-i}(x)\right\}-
$$

This shows that

$$
\beta(\beta(x))=x,
$$

and it follows from Theorem 9 that $x \leqq y$ if and only if $\beta(x) \geqq \beta(y)$. Hence the dual lattice $L^{\prime}$ is isomorphic with the lattice $L$, and in particular $L^{\prime}$ is a distributive lattice satisfying Axioms 1,2 , and 3.

Since $\left\{D_{n-i}(x)\right\}^{-}=\mathrm{V}_{j=0}^{n-1-1} C_{j}(x)$ and

$$
\bigvee_{i=1}^{n-1} e_{i}\left(\bigvee_{j=0}^{n-i-1} C_{j}(x)\right)=\bigvee_{j=0}^{n-2} C_{j}(x)\left(\bigvee_{i=1}^{n-j-1} e_{i}\right)=\bigvee_{j=0}^{n-2} C_{j}(x) e_{n-j-1}
$$

it follows that

$$
\beta(x)=\bigvee_{i=1}^{n-1} e_{i} C_{n-i-1}(x)
$$

Hence $e_{i}^{\prime}=\beta\left(e_{i}\right)=e_{n-i-1}$ by Theorem 4 , and (1) is proven. Then by the uniqueness theorem $C_{i}(\beta(x))=C_{n-i-1}(x)$. If $b \in B$, then by the Corollary of Theorem $3 C_{i}(\beta(b))=C_{n-i-1}(b)=0$ for $1 \leqq i \leqq n-2$ and $C_{n-1}(\beta(b))=C_{0}(b)=b$. Thus (6) yields $\beta(b)=b$. Note also that the set of complemented elements of $L^{\prime}$ is identical with the set $B$ of complemented elements of $L$. Now $\beta\left(C_{i}(x)\right)$ $=C_{i}^{\prime}(\beta(x))$ by the isomorphism, so that $C_{i}^{\prime}(x)=\beta\left(C_{i}(\beta(x))\right)=\left\{C_{i}(\beta(x))\right\}-$ $=\left\{C_{n-i-1}(x)\right\}-$ for $i=0,1, \cdots, n-1$, and (2) is proven. Similarly, using (4), $D_{i}^{\prime}(x)=\beta\left(D_{i}(\beta(x))\right)=\left\{D_{i}(\beta(x))\right\}^{-}=D_{n-i}(x)$, and this completes the proof.

Since $\beta(x \vee y)=\beta(x) \beta(y)$ and $\beta(x y)=\beta(x) \vee \beta(y)$, it is now easy to obtain the dual form of Axiom 3. In particular, $x=\beta(\beta(x))=\beta\left(\bigvee_{i=1}^{n-1} e_{i} C_{n-i-1}(x)\right)$, so that 


$$
x=\bigwedge_{i=1}^{n-1}\left(\beta\left(e_{i}\right) \vee \beta\left(C_{n-i-1}(x)\right)\right)=\bigwedge_{i=1}^{n-1}\left(e_{n-i-1} \vee\left\{C_{n-i-1}(x)\right\}-\right) ;
$$

that is, $x=\left\{C_{0}(x)\right\}^{-}\left(e_{1} \vee\left\{C_{1}(x)\right\}^{-}\right) \cdots\left(e_{n-2} \vee\left\{C_{n-2}(x)\right\}^{-}\right)$.

Theorem 11. For each $i=1,2, \cdots, n-1$ and for every $x \in L$ and $y \in L$, $D_{i}(x y)=D_{i}(x) D_{i}(y)$.

Proof. This is an easy consequence of Theorem 8 and the duality theorem. Specifically, for each $i=1,2, \cdots, n-1$, by Theorem $8, D_{n-i}(\beta(x) \bigvee \beta(y))$ $=D_{n-i}(\beta(x)) \bigvee D_{n-i}(\beta(y))$. That is, $D_{n-i}(\beta(x y))=D_{n-i}(\beta(x)) \bigvee D_{n-i}(\beta(y))$, so that by (4) of Theorem 10, $\left\{D_{i}(x y)\right\}^{-}=\left\{D_{i}(x)\right\}^{-} \vee\left\{D_{i}(y)\right\}-$, and so $D_{i}(x y)$ $=D_{i}(x) D_{i}(y)$.

TheOREM 12. The following identities are valid for each $i=0,1, \cdots, n-1$.

$$
\begin{aligned}
C_{i}(x \vee y) & =C_{i}(x) \bigvee_{j=0}^{i} C_{j}(y) \vee C_{i}(y) \bigvee_{j=0}^{i} C_{j}(x) . \\
C_{i}(x y) & =C_{i}(x) \bigvee_{j=i}^{n-1} C_{j}(y) \vee C_{i}(y) \bigvee_{j=i}^{n-1} C_{j}(x) .
\end{aligned}
$$

Proof. These identities are obtained easily through the use of the preceding theorems. Specifically,

$$
\begin{aligned}
C_{i}(x \vee y) & =D_{i}(x \vee y)\left\{D_{i+1}(x \vee y)\right\}- \\
& =\left(D_{i}(x) \vee D_{i}(y)\right)\left\{D_{i+1}(x)\right\}-\left\{D_{i+1}(y)\right\}^{-} \\
& =C_{i}(x)\left\{D_{i+1}(y)\right\}-\bigvee C_{i}(y)\left\{D_{i+1}(x)\right\}- \\
& =C_{i}(x) \bigvee_{j=0}^{i} C_{j}(y) \vee C_{i}(y) \bigvee_{j=0}^{i} C_{j}(x) .
\end{aligned}
$$

Similarly,

$$
\begin{aligned}
C_{i}(x y) & =D_{i}(x y)\left\{D_{i+1}(x y)\right\}- \\
& =D_{i}(x) D_{i}(y)\left(\left\{D_{i+1}(x)\right\}^{-} \vee\left\{D_{i+1}(y)\right\}^{-}\right) \\
& =C_{i}(x) D_{i}(y) \vee C_{i}(y) D_{i}(x) \\
& =C_{i}(x) \bigvee_{j=i}^{n-1} C_{i}(y) \vee C_{i}(y) \bigvee_{j=i}^{n-1} C_{i}(x) .
\end{aligned}
$$

A more general form of this theorem is given by Theorem 25 in $\$ 7$.

4. Post functions. The reduction of a Post function to a given form may well be a ponderous chore. It should be noted that the work of this section on canonical expansions and simplifications of Post functions is of an extremely facile nature.

Definition 3. A Post function of $m$ variables is a function which can be 
obtained from the constant functions $E_{i}\left(x_{1}, \cdots, x_{m}\right)=e_{i}$ and identity functions $I_{j}\left(x_{1}, \cdots, x_{m}\right)=x_{j}$ by a finite number of the operations $\vee, \wedge, C_{0}$, $C_{1}, \cdots, C_{n-1}$.

Theorem 13. If $f$ is a Post function of $m$ variables $x_{1}, \cdots, x_{m}$, then

$$
f\left(x_{1}, \cdots, x_{m}\right)=\bigvee_{0 \leq i_{j} \leqq n-1} f\left(e_{i_{1}}, \cdots, e_{i_{m}}\right) C_{i_{1}}\left(x_{1}\right) \cdots C_{i_{m}}\left(x_{m}\right) .
$$

Proof. There are $n^{m}$ terms of the form $C_{i_{1}}\left(x_{1}\right) C_{i_{2}}\left(x_{2}\right) \cdots C_{i_{m}}\left(x_{m}\right)$, and these are called the fundaments of the $m$ variables. It follows from Axiom 1 that

(1) distinct fundaments are disjoint, and

(2) the supremum of all the fundaments is $u$; in fact, the infimum of two distinct fundaments must include an infimum of the form $C_{i}\left(x_{j}\right) C_{k}\left(x_{j}\right)=0$, where $i \neq k$ for some $j$, and

$$
\bigvee_{0 \leq i_{j} \leq n-1} C_{i_{1}}\left(x_{1}\right) \cdots C_{i_{m}}\left(x_{m}\right)=\bigwedge_{j=1}^{m}\left(\bigvee_{i_{j}=0}^{n-1} C_{i_{j}}\left(x_{j}\right)\right)=\bigwedge_{j=1}^{m}(u)=u .
$$

It follows from (2) that the theorem is true for the constant functions $E_{i}$, and using Axiom 3, for the identity functions $I_{j}$. It is clear that if $f$ and $g$ satisfy the theorem, then $f \bigvee g$ satisfies the theorem. Using (1), it is also clear that $f g$ satisfies the theorem.

Now suppose $f$ is a Post function satisfying the theorem. Each of the terms $f\left(e_{i_{1}}, \cdots, e_{i_{m}}\right)$ is equal to one of the $e_{j}, 0 \leqq j \leqq n-1$, by Axiom 2a and Theorem 4 . Therefore

$$
f\left(x_{1}, \cdots, x_{m}\right)=\bigvee_{k=0}^{n-1} e_{k} T_{k}
$$

where $T_{k}=\mathrm{V} C_{i_{1}}\left(x_{1}\right) \cdots C_{i_{m}}\left(x_{m}\right)$, the supremum being extended over all $\left(i_{1}, \cdots, i_{m}\right)$ for which $f\left(e_{i_{1}}, \cdots, e_{i_{m}}\right)=e_{k}$. Using (1) and (2), the uniqueness theorem shows that $C_{k}\left(f\left(x_{1}, \cdots, x_{m}\right)\right)=T_{k}$. But

$$
\mathrm{V}_{0 \leq i_{j} \leq n-1} C_{k}\left(f\left(e_{i_{1}}, \cdots, e_{i_{m}}\right)\right) C_{i_{1}}\left(x_{1}\right) \cdots C_{i_{m}}\left(x_{m}\right)=T_{k},
$$

by Theorem 4 . Hence $C_{k}\left(f\left(x_{1}, \cdots, x_{m}\right)\right)$ satisfies the theorem. This completes the proof.

Theorem 14. If $f$ is a Post function of $m$ variables, then $f\left(x_{1}, \cdots, x_{m}\right)=\bigwedge_{0 \leq i_{j} \leq n-1}\left(f\left(e_{i_{1}}, \cdots, e_{i_{m}}\right) \vee\left\{C_{i_{1}}\left(x_{1}\right)\right\}-\vee \cdots \vee\left\{C_{i_{m}}\left(x_{m}\right)\right\}^{-}\right)$.

Proof. The statement follows from the preceding theorem and the duality theorem.

The $n^{m}$ terms of the form $\left\{C_{i_{1}}\left(x_{1}\right)\right\}-\vee \cdots \vee\left\{C_{i_{m}}\left(x_{m}\right)\right\}-$ are called the dual fundaments. The infimum of all the dual fundaments is 0 , and the 
supremum of two distinct dual fundaments is $u$-these properties following from the duality theorem applied to (1) and (2) of Theorem 13.

The canonical forms provide a starting point for the simplification of Post functions. The simplification of such functions is greatly aided by contemporary work on the analogous problem in Boolean algebra. In particular, methods described by W. V. Quine [8], the Harvard Computation Laboratory [2], M. Karnaugh [4], and R. H. Urbano and R. K. Mueller [11] can all be easily modified to apply to the more general case of simplification of the lattice theoretic Post functions defined above.

The simplification and manipulation of these functions is further eased by the fact that the fundaments and dual fundaments are composed of elements belonging to the Boolean algebra $B$. Such elements are therefore subject to familiar identities. As a specific illustration, consider the case $n=3$ and the simplifications of the function

$$
\begin{aligned}
f(x, y) & =C_{0}(x) \vee C_{1}(x) C_{2}(y) \vee C_{2}(x) C_{2}(y) \vee e_{1} C_{1}(y) \\
& =C_{0}(x) \vee\left\{C_{0}(x)\right\}-C_{2}(y) \vee e_{1} C_{1}(y) \\
& =C_{0}(x) \vee C_{2}(y) \vee e_{1} C_{1}(y) \\
& =C_{0}(x) \vee y .
\end{aligned}
$$

Let $f$ be a function in canonical form, such as $f=\mathrm{V}_{i=0}^{n-1} e_{i} C_{i}(f)$, where each $C_{i}(f)$ is expressed as a supremum of fundaments. It is possible, then, to simplify $f$ by inspection. More systematically, each $C_{i}(f)$ can be simplified by the modified form of one of the methods mentioned above. The resulting form for $f$ then provides a simplification in terms of the variables $e_{i}$ and $C_{i}\left(x_{j}\right), i=0, \cdots, n-1$ and $j=1, \cdots, m$. If a simplification is required which allows presence of the variables $x_{j}, j=1, \cdots, m$, then this form must be further manipulated, with Axiom 3, to provide a final simplification.

The exact modifications required for each of the above methods are obvious, and need not be elaborated here. The Boolean fundaments are replaced with the fundaments defined herein, and the work in general is effected to the base $n$ instead of the base 2 .

In Boolean algebra the above methods achieve varying degrees of success, and their relative merits in Post algebra are approximately the same. The "visual recognition" advantage of Karnaugh's map method is emphasized if the final form is to be expressed utilizing the variables $x_{1}, \cdots, x_{m}$. The topological method such as outlined by Urbano and Mueller has aroused some recent interest [10]. The figure in the following section illustrates the cube associated with simplifications of Post functions in three variables.

5. Examples. If $p$ is a fixed prime, let $R$ be a $p$-ring with unit. The ring $R$ is a commutative ring of characteristic $p$ such that $x^{p}=x$ for every $x \in R$. A ring theoretic function $f(x, y)$ in $R$ is determined by the $p^{2}$ values of $f(i, j)$, where $i=0,1, \cdots, p-1$ and $j=0,1, \cdots, p-1$. Let $M(x, y)$ and $m(x, y)$ be those ring theoretic functions for which $M(i, j)=\max (i, j)$ and $m(i, j)$ 
$=\min (i, j)$, where $i=0,1, \cdots, p-1$ and $j=0,1, \cdots, p-1$. Thus, for example, in the case $p=3$,

$$
\begin{aligned}
& M(x, y)=x+y+x^{2} \cdot y+2 \cdot x \cdot y+x^{2} \cdot y^{2}+x \cdot y^{2}, \\
& m(x, y)=2 \cdot x^{2} \cdot y+x \cdot y+2 \cdot x^{2} \cdot y^{2}+2 \cdot x \cdot y^{2},
\end{aligned}
$$

where the operations involved are the ring operations of addition and multiplication.

It is now a straightforward task to show that if $n=p$, then $R$ forms a lattice $L$, where $x \vee y=M(x, y), x y=m(x, y), e_{i}=i$ and $C_{i}(x)=\prod_{j \neq p-i}(x+j)$, $i=0,1, \cdots, p-1$, the lattice theoretic functions on the left being given in terms of the ring theoretic functions on the right.

Another important example is that of a lattice of $n$-valued functions which assume the values $0,1, \cdots, n-1$ on a set $S$, ordered in the usual way, and containing the $n$ constant functions $e_{i}$ of value $i$ and for each $f$ the $n$ functions $C_{i}(f), i=0, \cdots, n-1$, where $C_{i}(f)$ is that function which has the value $n-1$ on those points of $S$ where $f$ has the value $i$, and the value 0 on the other points of $S$. It is easily verified that Axioms 1, 2, and 3 are satisfied.

The figure on the following page is a Hasse diagram of the free 3-ring with one generator. The points are identified according to the ordering noted above. It is clear that this is also the lattice of all 3 -valued functions on a three point set. The eight darkened points in the diagram are the points of $B$.

6. Representation theory. The work of this section shows, among other things, that the lattice $L$ is equivalent to a Post algebra of order $n$. This equivalence can of course be proved by tedious calculations which do not make use of the representation theorems.

Definition 4. If $B$ is represented as a Boolean algebra of subsets of a set $S$, where the image of $b \in B$ is given by $\lambda(b) \subset S$, then an $n$-valued $B$-measurable function on $S$ is an $n$-valued function on $S$ with the property that for each $i=0,1, \cdots, n-1$ there exists $b_{i} \in B$ such that $f^{-1}(i)=\lambda\left(b_{i}\right)$.

TheOREM 15. If $B$ is represented as a Boolean algebra of a certain class of subsets of a set $S$, then $L$ is isomorphic with the set of all $n$-valued B-measurable functions on $S$.

Proof. If the image of $b \in B$ is given by $\lambda(b) \subset S$, then for $x \in L$, let $x$ correspond to the function $f$ such that $f=i$ on $\lambda\left(C_{i}(x)\right)$ for each $i=0,1, \cdots$, $n-1$. This correspondence is one-to-one, for if $x \neq y$, then $C_{i}(x) \neq C_{i}(y)$ for some $i$, and $\lambda\left(C_{i}(x)\right) \neq \lambda\left(C_{i}(y)\right)$.

Now let $f$ be the function corresponding to $x \in L$ and let $g$ be the function corresponding to $y \in L$. Suppose $i \leqq j$ and $P \in \lambda\left(C_{i}(x)\right), P \in \lambda\left(C_{j}(y)\right)$. Then $P \in \lambda\left(C_{j}(y) C_{i}(x)\right)$, and therefore $P \in \lambda\left(C_{j}(x \bigvee y)\right)$ by Theorem 12. Hence $x \vee y$ corresponds to $f \bigvee g \equiv \max (f(P), g(P))$ for each $P \in S$.

Finally, the correspondence is onto the set of all $n$-valued $B$-measurable functions on $S$. For let $f$ be such a function. Then there exists $b_{i} \in B$ such that $\lambda\left(b_{i}\right)=f^{-1}(i)$ for each $i=0,1, \cdots, n-1$. Since $\lambda\left(b_{i}\right) \cap \lambda\left(b_{j}\right)=\varnothing$, the empty 


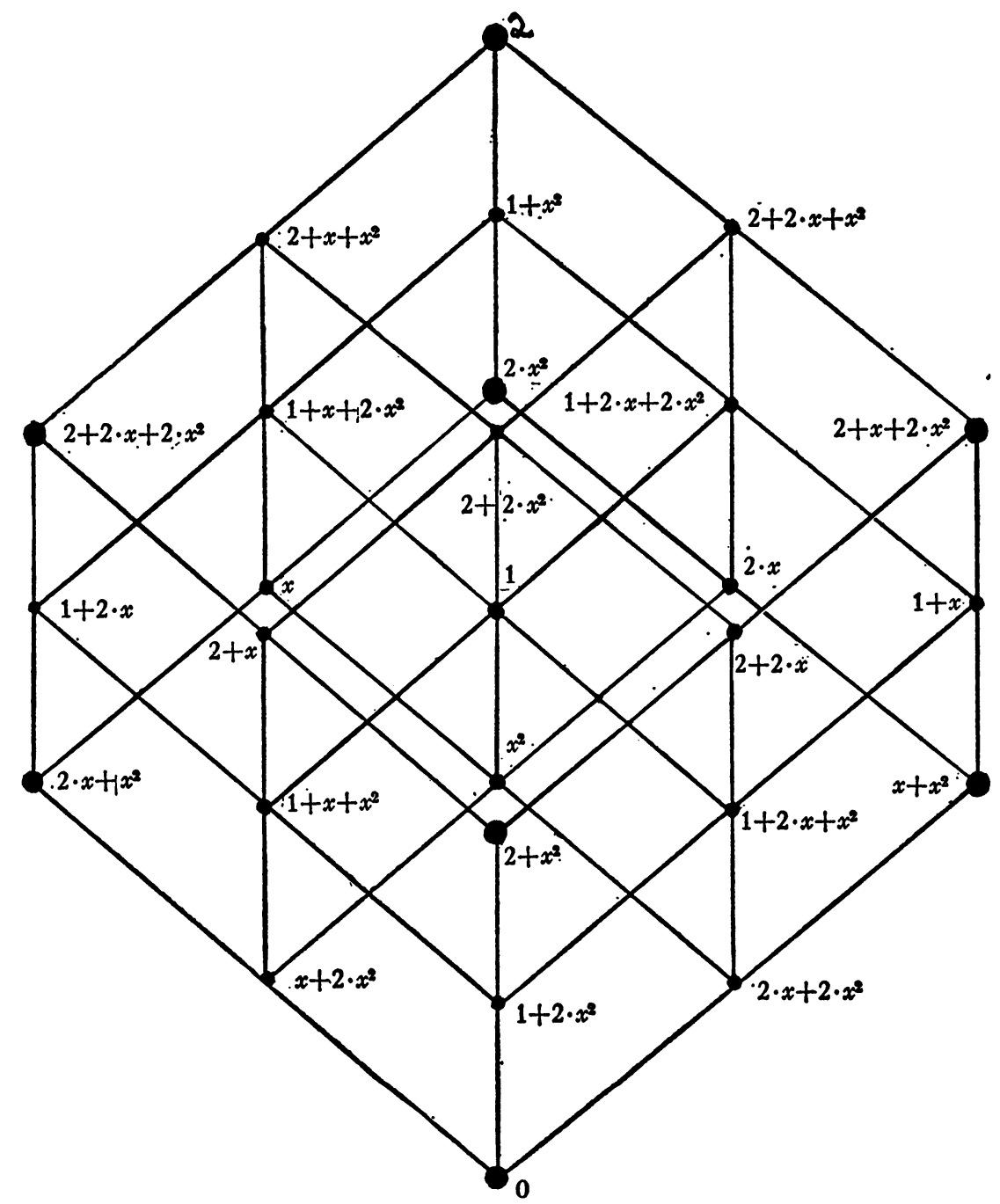

set, for $i \neq j$, and $\bigcup_{i=0}^{n-1} \lambda\left(b_{i}\right)=S, b_{i} b_{j}=0$ for $i \neq j$ and $V_{i=0}^{n-1} b_{i}=u$. Let $x=\bigvee_{i=1}^{n-1} e_{i} b_{i} \in L$. By the uniqueness theorem $C_{i}(x)=b_{i}$ for $i=0,1, \cdots, n-1$, so that $f$ is the function corresponding to $x$.

THEOREM 16. The lattice $L$ is isomorphic with the set of all continuous $n$ valued functions on a totally disconnected compact Hausdorff space.

Proof. This follows from the preceding theorem. It is known that $B$ can be represented as the algebra of open and closed sets in its representation space, and in this case the $n$-valued $B$-measurable functions are exactly the continuous $n$-valued functions.

In particular, $e_{i}$ corresponds to the constant function of value $i, i$ 
$=0,1, \cdots, n-1$, and, if $x$ corresponds to $f$, then $C_{i}(x)$ corresponds to the function $C_{i}(f)$ which has the value $n-1$ on those points of $S$ for which $f=i$, and has the value 0 on the other points of $S$. This, together with the latter example of the preceding section, leads to the following theorem.

Theorem 17. The lattice $L$ is a Post algebra of order $n$, and any Post algebra of order $n$ is such a lattice.

Proof. P. C. Rosenbloom [9] based his axiom system for Post algebras on the operation of "prime," which is defined for $n$-valued functions on a set $S$ as follows. If $f$ is such a function, then $f^{(\prime)}$ is that function which, if $f$ has the value $i$ at $P \in S$, has the value $i+1$ at $P$ if $i=0,1, \cdots, n-2$, and has the value 0 if $f$ has the value $n-1$ at $P$.

The lattice of functions described in the example of the preceding section is clearly closed under the operation of prime, since

$$
f^{(\prime)}=e_{1} C_{0}(f) \vee e_{2} C_{1}(f) \vee \cdots \vee e_{n-2} C_{n-3}(f) \vee C_{n-2}(f)
$$

Conversely, a lattice of $n$-valued functions on a set $S$ which is closed under the operation of prime must also include the constant functions $e_{i}$ and the functions $C_{i}(f), i=0,1, \cdots, n-1$. This follows from the easily verified formulas

$$
e_{i}=\left(\bigvee_{j=0}^{n-1} f^{(j)}\right)^{(i+1)} \quad \text { for any } f
$$

and

$$
C_{i}(f)=\left(\underset{j \neq n-i-1}{\vee} f^{(j)}\right)^{(\prime)},
$$

where $f^{(j)}$ is defined inductively by $f^{(i+1)}=\left(f^{(j)}\right)^{(\prime)}$.

Since P. C. Rosenbloom [9] and L. I. Wade [12] have shown that Rosenbloom's axioms for Post algebras of order $n$ describe lattices of $n$-valued functions closed under prime, the stated equivalence then follows.

Hence results obtained so far are equally valid for Post algebras of order $n$. Thus, in particular, Theorem 15 led to the representation of Post algebras given by Theorem 16. The following theorem gives a further result along these lines.

THEOREM 18. The lattice $L$ is complete and atomistic if and only if $L$ is isomorphic with the set of all $n$-valued functions on a set $S$.

Proof. It is clear from Theorem 16 that the set $E$ of elements less than or equal to $e_{1}$ is isomorphic with $B$ under the correspondence $b \rightarrow e_{1} b$. If $L$ is complete and atomistic, then $E$ is complete and atomistic, and hence is isomorphic with the class of all subsets of the set of all atoms of $E$. Hence $B$ is 
isomorphic with the class of all subsets of a set $S$, every $n$-valued function on $S$ is $B$-measurable, and the result follows by Theorem 15 . The converse is obvious.

In what follows, all prime ideals are nonzero and proper.

THEOREM 19. Every maximal chain of properly ascending prime ideals in $L$ consists of exactly $n-1$ prime ideals, and every prime ideal is a member of exactly one such chain.

Proof. The proof of Kaplansky's Theorem [3] shows that every prime ideal of the representation space given by Theorem 16 is associated with exactly one point of the representation space. That is, if $S$ denotes now the totally disconnected compact Hausdorff space, $P$ is a prime ideal of $L$, and $f$ and $g$ are continuous $n$-valued functions on $S$, then there exists exactly one point $p \in S$ with the property that if $f$ is a member of $P$ and if $g \leqq f$ in some neighborhood $N_{p}$ of $p$, then $g$ is also a member of $P$. However $g \leqq f$ at $p$ if and only if $g \leqq f$ in some neighborhood $N_{p}$, for if $g(p)=i$ and $f(p)=j$, let $N_{p}=g^{-1}(i) \cap f^{-1}(j)$, and the converse is obvious. Hence every prime ideal is of the form $P_{p i}=\{f \mid f(p) \leqq i-1\}$, where $i=1,2, \cdots, n-1$ and $p \in S$. Moreover, if $p$ and $p_{0}$ are different points of $S$, then there exists $b \in B$ with $p_{0} \notin \lambda(b)$ and $p \in \lambda(b)$, where $\lambda(b)$ is the open and closed subset of $S$ which is the image of $b$. Therefore if $b$ corresponds to the function $f$, then $f(p)=n-1$ and $f\left(p_{0}\right)=0$, since $p \in \lambda\left(C_{n-1}(b)\right)=\lambda(b)$ and $p_{0} \in \lambda\left(C_{0}(b)\right)=\lambda(b)$. Hence $P_{p_{0} i} \llbracket P_{p j}$ for $p \neq p_{0}$ and any $i=1,2, \cdots, n-1$ and $j=1,2, \cdots, n-1$. This completes the proof.

It follows from this argument that

$$
\left\{e_{i}\right\}=\bigcap_{p \in S}\left(P_{p(i+1)}-P_{p i}\right), \quad i=0,1, \cdots, n-1,
$$

where $P_{p 0}$ stands for the empty set and $P_{p n}$ stands for the entire set of functions in the representation space. For it is clear that $e_{i} \in P_{p(i+1)}-P_{p i}$ for each $p \in S, i=0,1, \cdots, n-1$. Now, for any $i(p)$, suppose that

$$
f \in \bigcap_{p \in S}\left(P_{p(i(p)+1)}-P_{p i(p)}\right) \text { and } g \in \bigcap_{p \in S}\left(P_{p(i(p)+1)}-P_{p i(p)}\right) .
$$

Then, if $f \neq g$, there exists a prime ideal $P$ which contains $g$ but not $f$. By Theorem 19 the prime ideal $P$ is of the form $P=P_{p_{0} i_{0}}$ for some $p_{0} \in S$ and some $i_{0}=1,2, \cdots, n-1$. This contradicts

$$
f \in P_{p_{0}\left(i\left(p_{0}\right)+1\right)}-P_{p_{0} i\left(p_{0}\right)} \text { and } g \in P_{p_{0}\left(i\left(p_{0}\right)+1\right)}-P_{p_{0} i\left(p_{0}\right)} \text {. }
$$

Hence there is at most one element in $\bigcap_{p \in S}\left(P_{p(i(p)+1)}-P_{p i(p)}\right)$ for any $i(p)$. Thus, in particular, for the constant values of $i(p),\left\{e_{i}\right\}=\bigcap_{p \in S}\left(P_{p(i+1)}-P_{p i}\right)$ for each $i=0,1, \cdots, n-1$.

These remarks lead to the following characterization of finite Post algebras of order $n$. 
TheOREM 20. Let $L_{D}$ be a finite distributive lattice in which every maximal chain of properly ascending prime ideals consists of exactly $n-1$ prime ideals, and every prime ideal is a member of exactly one such chain. Then $L_{D}$ is a Post algebra of order $n$.

Proof. Denote the chains of prime ideals by $P_{p 1} \subset P_{p 2} \subset \cdots \subset P_{p(n-1)}$, where $p$ ranges over some index set $S$. Let $P_{p 0}$ denote the empty set $\varnothing$ and $P_{p n}$ denote the entire set $L_{D}$. Then for every prime ideal $P \subset L_{D}$ there exists one and only one $p \in S$ and $i$ such that $P=P_{p i}$.

For any $x \in L_{D}$ and any $p \in S$ there exists $i(p, x)=i$ such that

$$
x \in P_{p(i+1)}-P_{p i} \text {. }
$$

Hence $x \in \bigcap_{p \in S}\left(P_{p(i(p, x)+1)}-P_{p i(p, x)}\right)$. Since such an intersection has at most one point, by the remarks preceding this theorem,

$$
\{x\}=\bigcap_{p \in S}\left(P_{p(i+1)}-P_{p i}\right) .
$$

If $y \in L_{D}$ and $j(p, y)=j$, so that $\{y\}=\bigcap_{p \in S}\left(P_{p(j+1)}-P_{p j}\right)$, it is easy to see that

$$
\{x \vee y\}=\bigcap_{p \in S}\left(P_{p(\max (i, j)+1)}-P_{p(\max (i, j))}\right)
$$

and

$$
\{x y\}=\bigcap_{p \in S}\left(P_{p(\min (i, j)+1)}-P_{p(\min (i, j))}\right),
$$

since the element at the left is contained in each of the sets in the intersection at the right.

On the other hand, if $L_{D}$ is finite, then for any $i(p), \bigcap_{p \in S}\left(P_{p(i(p)+1)}-P_{p i(p)}\right)$ $\neq \varnothing$. To prove this, note the following lemma.

Lemma 4. If $p_{1} \neq p_{2}, p_{1} \in S$ and $p_{2} \in S$, then $\left(P_{p_{1}(i+1)}-P_{p_{1} i}\right) \cap P_{p_{2} k} \neq \varnothing$ for any $i=0,1, \cdots, n-1$ and any $k=1,2, \cdots, n$.

Proof. If $P_{p_{1}(i+1)}-P_{p_{1} i} \subset L-P_{p_{2} k}$, then taking the complement of both sides, $P_{p_{2} k} \subset P_{p_{1} i} \cup\left(L-P_{p_{1}(i+1)}\right)$, so that

$$
P_{p_{1}(i+1)} \cap P_{p_{2} k} \subset P_{p_{1}(i+1)} \cap\left(P_{p_{1} i} \cup\left(L-P_{p_{1}(i+1)}\right)\right)=P_{p_{1} i}
$$

Hence $P_{p_{1} i} \supset P_{p_{1}(i+1)}$ or $P_{p_{1} i} \supset P_{p_{2} k}$, contradicting the hypotheses of the theorem.

Now let $S_{m}$ be a set of cardinality $m$, where $S_{m} \subset S$. Since $L_{D}$ is finite, $S$ itself is finite. Now for any $i(p)=i$ and any $S_{m}, \bigcap_{p \in S_{m}}\left(P_{p(i+1)}-P_{p i}\right) \neq \varnothing$. It follows easily from Lemma 4 that this statement is true for $m=2$, so suppose it is true for $m=k$. The set $S_{k+1}$ has $k+1$ distinct subsets of the form $S_{k}$, $S_{k 1}, S_{k 2}, \cdots, S_{k(k+1)}$, each of these being obtained by deleting a particular point of $S_{k+1}$. Let $x_{j} \in \bigcap_{p \in S_{k j}}\left(P_{p(i+1)}-P_{p i}\right)$ and let $\omega_{k}=\bigvee_{m=1}^{k+1}\left(\bigwedge_{j \neq m} x_{j}\right)$. Then 
it is straightforward to verify that $\omega_{k} \in \bigcap_{p \in S_{k+1}}\left(P_{p(i+1)}-P_{p i}\right)$. Hence $\bigcap_{p \in S}\left(P_{p(i(p)+1)}-P_{p i(p)}\right) \neq \varnothing$ for any $i(p)$.

The theorem itself now follows, for it is easy to verify, using (1) and (2), that Axioms 1, 2, and 3 are satisfied, where

$$
\left\{e_{i}\right\}=\bigcap_{p \in S}\left(P_{p(i+1)}-P_{p i}\right)
$$

and

$$
\left\{C_{j}(x)\right\}=\bigcap_{x \in P_{p(j+1)-P_{p j}}}\left(L-P_{p(n-1)}\right) \cap \bigcap_{x \notin P_{p(j+1)-P_{p j}}} P_{p 1} .
$$

In the case where $L_{D}$ is infinite, it need not be true that

$$
\bigcap_{p \in S}\left(P_{p(i(p)+1)}-P_{p i(p)}\right) \neq \varnothing
$$

for arbitrary $i(p)$, where now $S$ also is infinite. C. C. Chang and A. Horn have proved a corresponding theorem for the case of an infinite lattice, the additional assumption being a hypothesis which requires the existence of the $e_{i}, i=0,1, \cdots, n-1$.

7. Completeness properties. This section is devoted to certain results on infinite Post algebras. The proofs are dependent, in large part, on the formulation in $\$ 2$.

THEOREM 21. If $b_{i} \in B$ and either of the quantities

$$
\bigvee_{B ; i \in I} b_{i}, \quad \bigvee_{L ; i \in I} b_{i}
$$

exists, then the other exists and the two are equal.

Proof. If $b=\bigvee_{B ; i \in I} b_{i}$ exists let $y \in L$ and $y \geqq b_{i}$ for all $i \in I$. Then $C_{n-1}(y)$ $\geqq C_{n-1}\left(b_{i}\right)=b_{i}$ for all $i \in I$ by Theorem 9 and the Corollary of Theorem 3 . Hence $C_{n-1}(y) \geqq b$, and $y \geqq C_{n-1}(y) \geqq b$.

Conversely, if $r=\mathrm{V}_{L ; i \in I} b_{i}$ exists, then likewise $C_{n-1}(r) \geqq C_{n-1}\left(b_{i}\right)=b_{i}$ for all $i \in I$. Hence $C_{n-1}(r) \geqq r$. Since $r \geqq C_{n-1}(r), r=C_{n-1}(r) \in B$.

CoROllary. If $b_{i} \in B$ and either of the quantities

$$
\bigwedge_{B ; i \in I} b_{i}, \quad \bigwedge_{L ; i \in I} b_{i}
$$

exists, then the other exists and the two are equal.

Proof. This follows from the duality theorem.

Theorem 22. If $x=\bigvee_{L ; i \in I} x_{i}$ exists, where $x_{i} \in L$ for all $i \in I$, then $\bigvee_{B ; i \in I} D_{j}\left(x_{i}\right)$ exists and $D_{j}(x)=\mathrm{V}_{B ; i \in I} D_{j}\left(x_{i}\right)$ for each $j=1,2, \cdots, n-1$. Conversely, if $\bigvee_{B ; i \in I} D_{j}\left(x_{i}\right)$ exists for each $j=1,2, \cdots, n-1$, where $x_{i} \in L$ for all $i \in I$, then 
$\bigvee_{L ; i \in I} x_{i}$ exists and is equal to the element $x$ which is determined by the formulas $D_{j}(x)=\bigvee_{B ; i \in I} D_{j}\left(x_{i}\right)$. The dual statements are also true.

Proof. If $x=\bigvee_{L ; i \in I} x_{i}$ exists, then $x \geqq x_{i}$ for all $i \in I$, so that $D_{j}(x) \geqq D_{j}\left(x_{i}\right)$ for all $i \in I$ and each $j=1,2, \cdots, n-1$, by Theorem 9. Secondly, suppose that $w \geqq D_{j}\left(x_{i}\right)$ for some fixed $j=1,2, \cdots, n-1$ and all $i \in I$. Let $w^{\prime}$ $=C_{n-1}(w) \bigvee e_{j-1}$. Then it follows from Theorem 12, and from Theorem 3 and Theorem 4 , that $C_{n-1}\left(w^{\prime}\right)=C_{n-1}(w)$, and also that $C_{i}\left(w^{\prime}\right)=0$ for $i=j, j+1$, $\cdots, n-2$. Hence

$$
D_{j}\left(w^{\prime}\right)=\bigvee_{i=j}^{n-1} C_{i}\left(w^{\prime}\right)=C_{n-1}(w) .
$$

It is clear from Axiom 3 that, for each $i \in I, x_{i} \leqq e_{j-1} \vee D_{j}\left(x_{i}\right)$. Since $w \geqq D_{j}\left(x_{i}\right)$, $C_{n-1}(w) \geqq C_{n-1}\left(D_{j}\left(x_{i}\right)\right)=D_{j}\left(x_{i}\right)$ by Theorem 9 and Theorem 3 . Hence $x_{i}$ $\leqq e_{j-1} \vee C_{n-1}(w)=w^{\prime}$ for all $i \in I$. Thus $x \leqq w^{\prime}$, and $D_{j}(x) \leqq D_{j}\left(w^{\prime}\right)=C_{n-1}(w)$ $\leqq w$.

For the converse $\bigvee_{B ; i \in I} D_{k}\left(x_{i}\right) \geqq \bigvee_{B ; i \in I} D_{j}\left(x_{i}\right) \in B$ for all $j$ and $k$ in the range $1 \leqq k \leqq j \leqq n-1$, so that

$$
\bigvee_{B ; i \in I} D_{j}\left(x_{i}\right)=D_{j}(x), \text { where } x=\bigvee_{j=1}^{n-1} e_{j}\left(\bigvee_{B ; i \in I} D_{j}\left(x_{i}\right)\right),
$$

by Theorem 7 . Hence $D_{j}(x) \geqq D_{j}\left(x_{i}\right)$ for all $i \in I$ and each $j=1,2, \cdots, n-1$, and by Theorem $9, x \geqq x_{i}$ for all $i \in I$. If $y \geqq x_{i}$ for all $i \in I$, then $D_{j}(y) \geqq D_{j}\left(x_{i}\right)$ for all $i \in I$ and each $j=1,2, \cdots, n-1$, so that $D_{j}(y) \geqq D_{j}(x)$ for each $j=1,2, \cdots, n-1$, and by Theorem 9 again, $y \geqq x$.

The dual statements are equally valid. This follows from the duality theorem, where it is shown that $D_{j}^{\prime}(x)=D_{n-j}(x)$ for each $j=1,2, \cdots, n-1$.

THEOREM 23. The lattice $L$ is complete if and only if $B$ is complete.

Proof. If $L$ is complete then $B$ is complete by Theorem 21. If $B$ is complete then $L$ is complete by Theorem 22 .

The infinite distributive law $y \bigvee_{i \in I} x_{i}=\bigvee_{i \in I} y x_{i}$ is valid in any Boolean algebra whenever $\mathrm{V}_{i \in I} x_{i}$ exists. This law is also valid in any complete pseudocomplemented lattice. Hence, by Theorem 5, this law (and its dual) is valid in $L$ whenever $L$ is complete. That is, if the Post algebra $L$ is complete, then $L$ is a topological lattice $[1$, p. 146]. However the next theorem shows that this infinite distributivity law holds in any Post algebra $L$.

THEOREM 24. The infinite distributive law $y \bigvee_{i \in I} x_{i}=\bigvee_{i \in I} y x_{i}$ is valid in any Post algebra $L$ whenever $x=\bigvee_{i \in I} x_{i}$ exists. The dual statement is also true.

Proof. By Theorem 22, for each $j=1,2: \cdots, n-1, D_{j}(x)=\mathrm{V}_{B ; i \in I} D_{j}\left(x_{i}\right)$. 
Thus

$$
D_{j}(y) D_{j}(x)=D_{j}(y) \underset{B_{i} i \in I}{\bigvee} D_{j}\left(x_{i}\right)=\bigvee_{B_{i} i \in I} D_{j}(y) D_{j}\left(x_{i}\right),
$$

since the law is valid in any Boolean algebra. Hence, by Theorem $11, D_{j}(y x)$ $=\mathrm{V}_{B ; i \in I} D_{j}\left(y x_{i}\right)$ for each $j=1, \cdots, n-1$, and $y x=\mathrm{V}_{L ; i \in I} y x_{i}$ by Theorem 22. The duality theorem yields the dual result.

TheOREM 25. If $x=\mathrm{V}_{i \in I} x_{i}$ exists, then the following generalized rules of simplification, stated in recursive form, are valid.

$$
C_{n-1}(x)=\bigvee_{i \in I} C_{n-1}\left(x_{i}\right) .
$$

$$
C_{k}(x)=\vee \bigvee_{i \in I}\left(\bigwedge_{j=k+1}^{n-1}\left\{C_{j}(x)\right\}^{-}\right) C_{k}\left(x_{i}\right) \quad \text { for } k=n-2, n-3, \cdots, 1,0 .
$$

Dually, if $y=\bigwedge_{i \in I} y_{i}$ exists, then

$$
C_{0}(y)=\bigvee_{i \in I} C_{0}\left(y_{i}\right) \text {. }
$$

(2)

$$
C_{k}(y)=\bigvee_{i \in I}\left(\bigwedge_{j=0}^{k-1}\left\{C_{j}(y)\right\}-\right) C_{k}\left(y_{i}\right), \quad \text { for } k=1,2, \cdots, n-2, n-1 .
$$

Proof. The first statement follows from Theorem 6 and Theorem 22 . Specifically, $C_{n-1}(x)=D_{n-1}(x)$, and for $k<n-1$,

$$
\begin{aligned}
C_{k}(x) & =D_{k}(x)\left\{D_{k+1}(x)\right\}^{-} \\
& =\left(\bigvee_{i \in I} D_{k}\left(x_{i}\right)\right)\left\{\bigvee_{i \in I} D_{k+1}\left(x_{i}\right)\right\}^{-} \\
& =\left(\bigvee_{i \in I} D_{k}\left(x_{i}\right)\right)\left(\bigwedge_{i \in I}\left\{D_{k+1}\left(x_{i}\right)\right\}^{-}\right) .
\end{aligned}
$$

Hence, by the infinite distributivity law,

$$
\begin{aligned}
C_{k}(x) & =\bigvee_{i \in I}\left(D_{k}\left(x_{i}\right) \bigwedge_{i \in I}\left\{D_{k+1}\left(x_{i}\right)\right\}-\right) \\
& =\bigvee_{i \in I}\left(C_{k}\left(x_{i}\right) \bigwedge_{i \in I}\left\{D_{k+1}\left(x_{i}\right)\right\}^{-}\right) \\
& =\bigvee_{i \in I} C_{k}\left(x_{i}\right)\left\{D_{k+1}(x)\right\}^{-} \\
& =\bigvee_{i \in I}\left(C_{k}\left(x_{i}\right) \bigwedge_{j=k+1}^{n-1}\left\{C_{j}(x)\right\}^{-}\right) .
\end{aligned}
$$


The second statement follows by the duality theorem, where it is shown that $C_{i}^{\prime}(x)=\left\{C_{n-i-1}(x)\right\}^{-}$. Applying this to (1) yields

$$
\begin{aligned}
&\left\{C_{0}(y)\right\}^{-}=\bigwedge_{i \in I}\left\{C_{0}\left(y_{i}\right)\right\}^{-} \\
&\left\{C_{n-k-1}(y)\right\}^{-}=\bigwedge_{i \in I}\left(\bigvee_{j=k+1}^{n-1} C_{n-j-1}(y) \bigvee\left\{C_{n-k-1}\left(y_{i}\right)\right\}-\right) \\
& \quad \text { for } k=n-2, n-3, \cdots, 1,0 .
\end{aligned}
$$

Then (2) follows from this by taking complements.

TheOREM 26. The normal completion $\left[1\right.$, p. 58] $L_{N}$ of a Post algebra is also a Post algebra. Furthermore $B_{N}$, the normal completion of $B$, is the Boolean algebra of complemented elements of $L_{N}$.

Proof. The lattice $L$ can be represented as the set of $n$-valued $B$-measurable functions on the representation space of $B$; that is, the set of functions $f$ such that $f^{-1}(i)$ for each $i=0,1, \cdots, n-1$ is an open and closed set. It is well known that $B_{N}$ is isomorphic with the algebra of regular open sets in the representation space of $B\left[1\right.$, p. 177] . Let $L_{N}$ be the set of $n$-valued $B_{N^{-}}$ measurable functions on the representation space of $B$; that is, the set of functions $f$ such that $f^{-1}(i)$ for each $i=0,1, \cdots, n-1$ is a regular open set. Then $L \subset L_{N}$ and, since $B_{N}$ is complete, $L_{N}$ is complete by Theorem 23 .

Now for any $x \in L_{N}$ it will be shown that there exist $x_{i} \in L, i \in I$, such that $x=V_{L_{N} ; i \in I} x_{i}$. Then by the duality theorem it will follow that there exist $y_{i} \in L, i \in I$, such that $x=\Lambda_{L_{N} ; i \in I} y_{i}$, and this will complete the proof that $L_{N}$ is the normal completion of $L$. If $x \in L_{N}$, there exist $C_{j}(x) \in B_{N}$, where $j=0,1, \cdots, n-1$, with $x=\mathrm{V}_{j=1}^{n-1} e_{j} C_{j}(x)$. Since $B_{N}$ is the normal completion of $B$, there exist $x_{j i} \in B$ such that $\bigvee_{B_{N} ; i \in I} x_{j i}=C_{j}(x) \in B_{N}$, for each $j=1,2, \cdots, n-1$. For each $i$ the quantities $x_{(n-1) i}, \cdots, x_{2 i}, x_{1 i}, \bigwedge_{j=1}^{n-1} \bar{x}_{j i}$ are pairwise disjoint and their supremum is $u$. Hence, by the uniqueness theorem, if $x_{i}=\mathrm{V}_{j=1}^{n-1} e_{j} x_{j i}$, then $C_{j}\left(x_{i}\right)=x_{j i}$ for each $j=1,2, \cdots, n-1$ and all $i \in I$. Now each of the quantities $\bigvee_{B_{N} ; i \in I} D_{j}\left(x_{i}\right)$ exist for $j=1,2, \cdots, n-1$, so that Theorem 22 yields $x=\mathrm{V}_{L_{N} ; i \in I} x_{i}$.

\section{BIBLIOGRAPHY}

1. G. Birkhoff, Lattice theory, rev. ed., New York, 1948.

2. Staff of Harvard Computation Laboratory, Synthesis of electronic computing and control circuits, Cambridge, 1951.

3. I. Kaplansky, Lattices of continuous functions, Bull. Amer. Math. Soc. vol. 53 (1947) pp. 617-622.

4. M. Karnaugh, The map method for synthesis of combinatorial logic circuits, Trans. Amer. Inst. Elec. Engrs. vol. 72 (1953) pp. 593-599.

5. N. H. McCoy, Rings and ideals, Buffalo, 1948. 
6. N. H. McCoy and D. Montgomery, A representation of generalized Boolean rings, Duke Math. J. vol. 3 (1937) pp. 455-459.

7. E. L. Post, Introduction to a general theory of elementary propositions, Amer. J. Math. vol. 43 (1921) pp. 163-185.

8. W. V. Quine, The problem of simplifying truth functions, Amer. Math. Monthly vol. 59 (1952) pp. 521-531.

9. P. C. Rosenbloom, Post algebras I. Postulates and general theory, Amer. J. Math. vol. 64 (1942) pp. 167-188.

10. J. P. Roth, Algebraic topological methods for the synthesis of switching systems, Trans. Amer. Math. Soc. vol. 88 (1958) pp. 301-326.

11. R. H. Urbano amd R. K. Mueller, $A$ topological method for the determination of the minimal forms of a Boolean function, I.R.E. Trans. on Elect. Computers vol. EC-5 (1956) pp. 126-132.

12. L. I. Wade, Post algebras and rings, Duke Math. J. vol. 12 (1945) pp. 389-395.

\author{
University OF California, \\ Los Angeles, California \\ Hughes Aircraft Company, \\ Culver City, California
}

